\title{
Exploiting optical properties of nanopolycrystalline diamond in high pressure experiments
}

\section{J. Antonio Barreda-Argüeso, Jesús González, Rafael Valiente, Tetsuo Irifune \& Fernando Rodríguez}

To cite this article: J. Antonio Barreda-Argüeso, Jesús González, Rafael Valiente, Tetsuo Irifune \& Fernando Rodríguez (2019): Exploiting optical properties of nanopolycrystalline diamond in high pressure experiments, High Pressure Research

To link to this article: https://doi.org/10.1080/08957959.2019.1702173

\section{曲 Published online: 17 Dec 2019.}

Submit your article to this journal 중

Q View related articles $₫$

View Crossmark data $\nearrow$ 


\title{
Exploiting optical properties of nanopolycrystalline diamond in high pressure experiments
}

\author{
J. Antonio Barreda-Argüeso ${ }^{a}$, Jesús González ${ }^{a}$, Rafael Valiente ${ }^{b}$, Tetsuo Irifune ${ }^{c}$ and \\ Fernando Rodríguez ${ }^{\mathrm{a}}$ \\ ${ }^{a}$ Facultad de Ciencias, Department Earth Sciences and Condensed Matter Physics (DCITMAC), University of \\ Cantabria, Santander, Spain; ${ }^{\mathrm{b}}$ Applied Physics Department, University of Cantabria \& IDIVAL, Santander, Spain; \\ 'Geodynamics Research Center, Ehime University, Matsuyama, Japan
}

\begin{abstract}
We investigated the optical properties (absorption, luminescence and Raman spectra) of nanopolycrystalline diamond (NPD) aiming at exploring its capabilities as a pressure sensor and as a pressurecell anvil for combined X-ray/neutron and optical studies. Notably, we analysed the Raman peak shift and broadening with pressure using a Moissanite Anvil Cell (MAC). The results are compared with those obtained in a DAC, where Raman signals from NPD chips and diamond anvils strongly overlap. Its pressure behaviour in the hydrostatic and non-hydrostatic regimes were investigated. We showed that the nanopolycrystalline structure induces remarkable differences in the peak shift and broadening between NPD and natural type lla single-crystal diamond, making NPD suitable as pressure gauge for pressure determination and testing hydrostaticity of pressure transmitting medium.
\end{abstract}

\section{ARTICLE HISTORY}

Received 1 September 2019 Accepted 3 December 2019

\section{KEYWORDS}

Raman spectroscopy;

luminescence; pressure gauge; pressure sensitivity; nanopolycrystalline diamond

\section{Introduction}

Since the first synthesis of nanopolycrystalline diamond (NPD) at high temperature (2300$2500 \mathrm{~K}$ ) and high pressure (15-25 GPa) [1], a wide variety of applications were foreseen by exploiting their unique structural and physical properties. Due to their isotropic enhanced hardness, NPD is expanding its use as anvil material in diamond anvil cell (DAC) in the realm of high pressure research. For instance, it came up with notably improvements in: X-ray absorption spectroscopy (XAS) suppressing the unavoidable presence of Bragg spots of single-crystal diamond due to its polycrystalline nature [3], laser-heating experiments in DAC where the lower thermal conductivity of NPD allows to reach higher temperatures exceeding $5000 \mathrm{~K}$ [4], or easy micro-anvil machinery to get extreme static pressures in double-stage DACs [5]. Among these applications, their genuine optical properties [6-8] have been less explored, probably because of their optical limitations compared to conventional DAC. Its reduced wavelength range in the UV-VIS and strong photoluminescence $(\mathrm{PL})$ precludes their use in pressure experiments dealing with the characterisation of optical properties using DACs.

CONTACT Fernando Rodríguez fernando.rodriguez@unican.es E Facultad de Ciencias, Department Earth Sciences and Condensed Matter Physics (DCITMAC), University of Cantabria, Santander 39005, Spain 
This work focuses on the optical characterisation of large NPD anvil for its eventual use in large volume anvil cells. The results will be crucial to determine the optical window and PL activity for the use of spectroscopy probes in NPD anvils, and their sensor capabilities for using as pressure gauge. In particular, we have studied the optical absorption, emission/excitation, and Raman spectra as a function of pressure and temperature. The latter is important for an eventual use of the NPD Raman mode at $1332 \mathrm{~cm}^{-1}$ as pressure probe [1,2]. Interestingly, we have measured the pressure shift of the NPD Raman line inside the pressure cavity using moissanite anvils [9]. The result allows us to compare the obtained pressure shifts with others measured on the culet of the diamond anvil, which are often used as pressure gauge. The variability of pressure coefficients reported in the literature for the Raman shift [10-18] makes the determination of pressure a rather controversial issue, even more if we consider both the difficulty to get the actual internal pressure from the Raman spectrum of the culet, and the dependence of the pressure coefficient with the anvil geometry [14].

\section{Experimental}

NPD samples ( $8 \mathrm{~mm}$ in thickness) were supplied by the Geodynamics Research Institute (Ehime University) under PRIUS collaborative program; $8 \mathrm{~mm}$-thick single-crystal chemical vapour deposition diamond (CVD) were obtained from WD Lab Grown Diamonds, and $3 \mathrm{~mm}$-thickness natural type lla diamonds (NatD) from Almax-easyLab Inc.

The optical absorption spectra were taken with a CARY $6000 i$ spectrophotometer in the 200-1800 nm range, and a Thermo Nicolet Continuum FTIR-spectrometer was used for the $400-4000 \mathrm{~cm}^{-1}$ range. For PL measurements, the excitation and emission spectra were obtained by means of a FLSP920 (Edinburgh Inst.) fluorescence spectrometer in the UVVIS-NIR. In all measurements, samples were placed in an excitation/emission configuration of $90^{\circ}$ and forward scattering. Spectra were recorded at room and low temperatures $(30 \mathrm{~K}$ for absorption and $10 \mathrm{~K}$ for emission/excitation).

Due to the strong sample absorption outside the transparency window (460$2500 \mathrm{~nm}$ ) we selected laser wavelengths within this window and kept the power of the laser beam below $4 \mathrm{~mW}$ to avoid heating of the sample. The Raman spectra were taken with a Horiba T64000 triple monochromator using the 488 and $514.5 \mathrm{~nm}$ lines of a Coherent Innova Spectrum $70 \mathrm{Ar}^{+}-\mathrm{Kr}^{+}$laser and nitrogen-cooled CCD (Jobin-Yvon Symphony) with a confocal microscope for detection. A Raman Glacier $x$ spectrometer was used to obtain Raman spectra under NIR excitation at $785 \mathrm{~nm}$. A membrane diamond anvil cell (DAC) and a moissanite membrane anvil cell (MAC) were used for high pressure Raman experiments. Both anvils were supplied by the Almax-easyLab Inc. 200- $\mu \mathrm{m}$-thick Inconel gaskets were pre-indented and suitable $150 \mu \mathrm{m}$ diameter holes were perforated with a BETSA motorised electrical discharge machine. Raman spectra of NPD under high pressure conditions were performed using methanolethanol (4:1) and spectroscopic paraffin oil (Merck) as pressure transmitting media. Pressure was calibrated using the fluorescence ruby method. 


\section{Results and discussions}

\section{Optical absorption}

Figure 1 compares the absorption spectrum of NPD with the corresponding spectra of NatD and CVD. The spectra are similar to those published elsewhere $[7,8]$ but thicker diamonds $(8 \mathrm{~mm})$ have been used in this work to allow precise comparison of the absorption features appearing just below the absorption threshold of NPD, which is located about $460 \mathrm{~nm}$. The spectra illustrate an important limitation of NPD for light transmission in the UV-blue range. Whereas NatD and CVD transmit light for wavelengths larger than their bandgap at $225 \mathrm{~nm}$, NPD is inappropriate for transmitting light below about $460 \mathrm{~nm}$. However, it must be noted that the transparency range is well suited for imaging from DAC equipped with NPD anvils. This allows a wide range of excitation wavelengths for Raman spectroscopy, although PL is produced in wide excitation range. The combination of absorption and excitation spectra associated to the red PL allows us to select the different optical windows for an appropriate Raman study (see next section). A salient feature of NPD with respect to CVD and NatD is the presence of a shoulder between 500 and $700 \mathrm{~nm}$, which is responsible for its characteristic brownish colour, together with specific absorption lines at 613 and $668 \mathrm{~nm}$, and two weak lines at 695 and $710 \mathrm{~nm}$ (Figure 1(a)). Aside from the weak features observed at 575 and $653 \mathrm{~nm}$, which are well-known nitrogen-related centres associated with neutral nitrogen-vacancy $\left(\mathrm{NV}^{0}\right)$ and negatively charged nitrogen-boron $\left(\mathrm{NB}^{-}\right)$centres [19], the origin of the specific NPD lines is still unclear. However, the idea that these lines could be associated with intrinsic centres, which are formed in the boundary region between nanodiamonds is likely $[7,8]$. The low-temperature PL spectra given below support this hypothesis. No absorption feature is observed in NPD between 800 and $2500 \mathrm{~nm}$, with the exception of CVD where weak peaks at $1359,1458,1556 \mathrm{~nm}$, which are associated with hydrogenrelated centres $[20,21]$, are observed in the absorption spectrum.
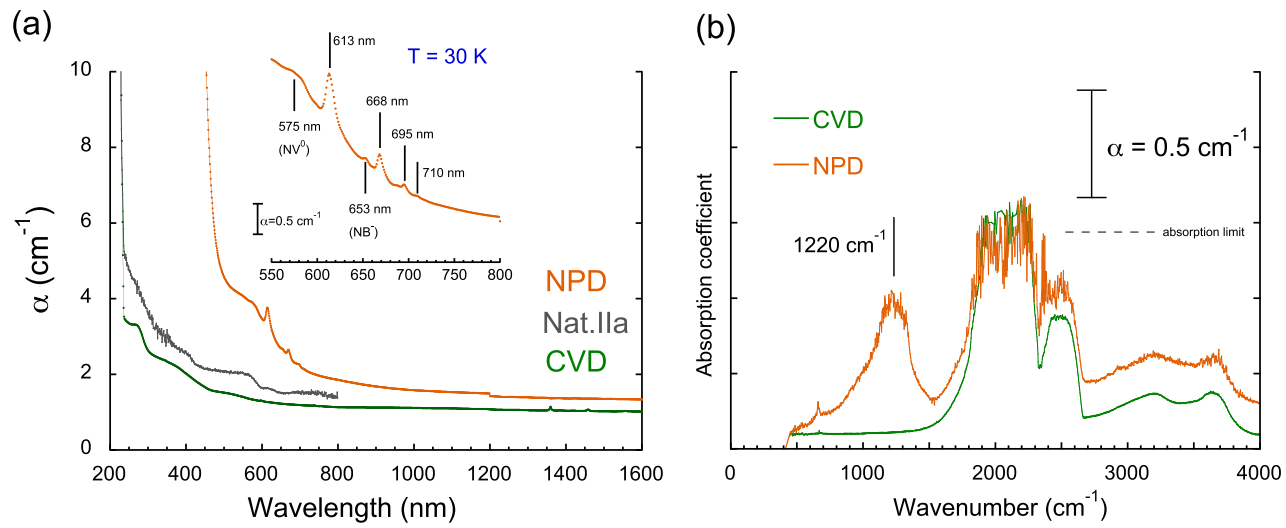

Figure 1. (a) Absorption spectra in the UV-VIS-NIR range of different diamonds: NPD, CVD and NatD. The inset shows a magnification of the absorption bump between 550 and $800 \mathrm{~nm}$ over which there are several absorption peaks. (b) FTIR absorption spectra in the $400-4000 \mathrm{~cm}^{-1}$ range. Dashed line indicates detection limit of FTIR-spectrometer. 
The IR absorption spectra show the characteristic multi-phonon absorption between 1500 and $4000 \mathrm{~cm}^{-1}$, which is a common feature of all diamonds [7]. The presence of the $1220 \mathrm{~cm}^{-1}$ band in NPD, which is absent in both CVD and NatD, is noteworthy. This band has been related to inclusions of hexagonal $6 \mathrm{H}$ diamond in the inter-nanograin region $[7,8,22]$. Although this hypothesis still deserves further experimental confirmation, Density-Functional-Theory calculations have shown that hexagonal $6 \mathrm{H}$ diamond has three IR-active optical phonons at $\Gamma$ point with frequency (symmetry) of $1218.7\left(E_{1 u}\right), 1197.5\left(A_{2 u}\right)$ and $1031.3 \mathrm{~cm}^{-1}\left(\mathrm{~A}_{2 \mathrm{u}}\right)$ [22], which fairly accounts for the observed NIR band and corresponding shoulder structure (Figure 1(b)). The diminution of the absorption coefficient of this band with the synthesis temperature from 2700 to $3300 \mathrm{~K}$ is consistent with the progressive transformation of the metastable hexagonal $6 \mathrm{H}$ diamond inclusions to cubic diamond [7].

\section{Photoluminescence spectra}

A remarkable characteristic of NPDs is their PL under excitation in a wide UV-VIS-NIR range. Figure 2(a) shows the emission spectra of the two samples, namely NPD 03488 and NPD03489, under excitation at $488 \mathrm{~nm}$. Besides the Raman sharp features at $521.9 \mathrm{~nm}$, the room-temperature PL spectra consists of a sample-dependent broad emission band centred between 550 and $650 \mathrm{~nm}$, with a complex structure of zero-phonon lines (ZPL) and associated phono-side bands, averaged over different PL centres formed during the high pressure high-temperature NPD synthesis, weighted by their relative abundance and PL efficiency. Hence, the emission band depends on the thermal history and is thus sample dependent. Interestingly, the Raman peak at $521.9 \mathrm{~nm}$ is slightly sample dependent as well (Figure 2(b)). Although their frequency is at $1331.9 \mathrm{~cm}^{-1}$ their frequency difference is within $0.2 \mathrm{~cm}^{-1}$ in both NPD samples-, the full width at

(a)

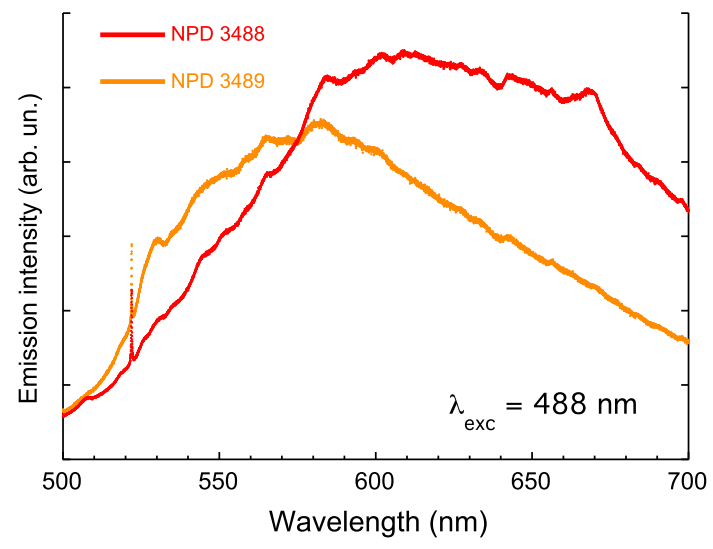

(b)

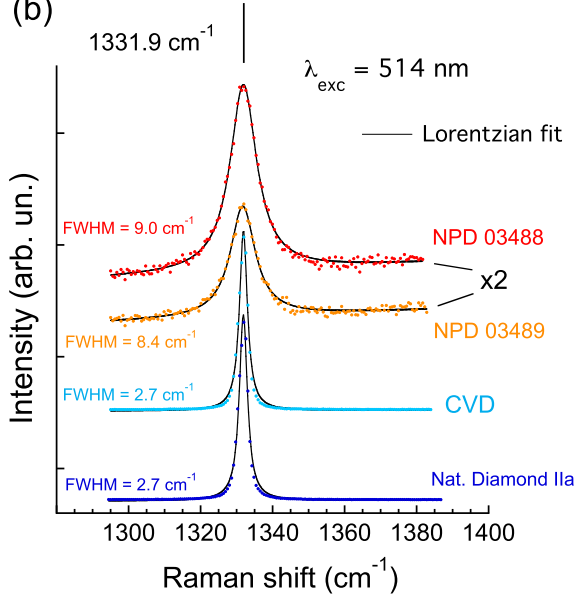

Figure 2. (a) Emission spectra of NPD samples under laser excitation at $488 \mathrm{~nm}$. Peaks at $521.9 \mathrm{~nm}$ correspond to the $1331.9 \mathrm{~cm}^{-1}$ phonon mode. (b) One-phonon Raman spectra of the two NPD samples, CVD and NatD. Continuous black lines correspond to the fitting of Raman peaks to Lorentzian functions. Fit parameters are $1331.9(3) \mathrm{cm}^{-1}$ for the peak maximum in all samples except for NPD03489 $\left(1331.7 \mathrm{~cm}^{-1}\right)$; FWHM values are indicated in the figure. 
half maximum (FWHM), though similar, is significantly different: 8.4 and $9.0 \mathrm{~cm}^{-1}$ for NPD 03489 and NPD03488, respectively. It must be noted that such a slight difference correlates with the emission broadband. The broader the Raman peak, the broader and more redshifted the emission band. Worthily, the Raman frequency is, within the spectral accuracy, the same for NPD, CVD, and NatD. The different FWHM of each diamond is discussed later on. This variation of the shape and structure of the room-temperature broadband emission has already been observed in nanodiamonds with nitrogen-impurity-related colour centres $[23,24]$. The emission band can be easily modified just changing the relative concentration of PL centres, as it was demonstrated for example by controlling the relative concentration of nitrogen centres $\mathrm{NV}^{0}$ and $\mathrm{NV}^{-}$in $\mathrm{PL}$ diamonds [24].

Figure 3 shows the low-temperature emission and excitation spectra in comparison with the absorption spectrum around the $\mathrm{PL}$ region. Both emission and excitation spectra consist of a mixed broadband-ZPL structure in the yellow-red spectral range, which are associated with different defects and impurities present in NPD. Major PL features of the emission spectra are related to ZPLs at 613 and $667 \mathrm{~nm}$, which are specific features of NPD [7,8]. Figure 3 shows how the sharp peaks observed in absorption coincides with ZPLs of the emission and excitation spectra, which were obtained exciting at the $612 \mathrm{~nm} \mathrm{ZPL}$, and by detecting the PL in the long-wavelength emission tail at $720 \mathrm{~nm}$, respectively. The excitation spectra mainly consist of ZPLs at 613 and $667 \mathrm{~nm}$,

(a)

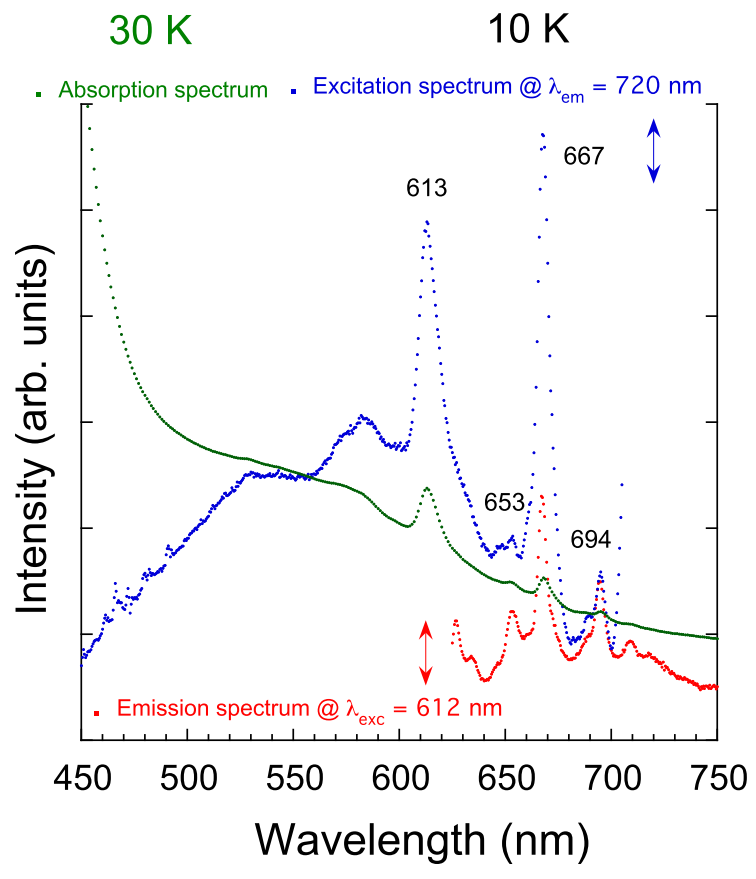

(b)

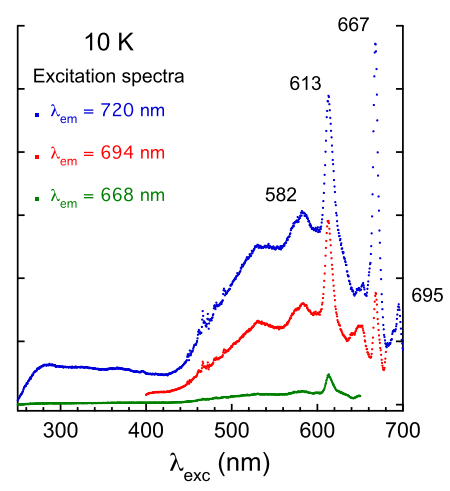

(c)

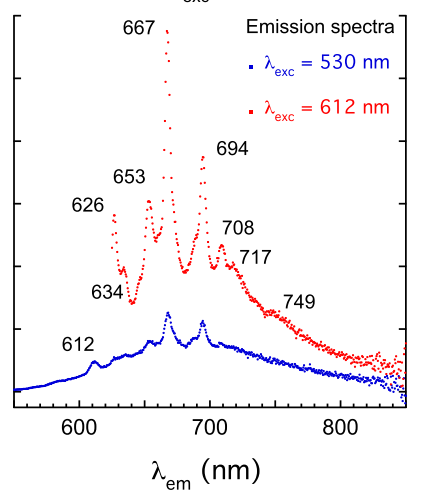

Figure 3. (a) Comparison of the low-temperature absorption, excitation and emission spectra of NPD. Detailed excitation (b) and emission (c) spectra at selected emission and excitation wavelengths, respectively. Spectral resolution: $0.5 \mathrm{~nm}$. Spectra were corrected for instrumental response. 
as the absorption peaks, thus suggesting that the NPD PL is related to specific NPD centres, which are probably formed in the nanodiamond boundary regions. The emission spectra under ZPL excitation at $612 \mathrm{~nm}$ show ZPLs at 653, 667 and $694 \mathrm{~nm}$, corresponding to different centres. It implies that, under excitation at $612 \mathrm{~nm}$, NPD emits not only from the $612 \mathrm{~nm}$ centre but also from other centres, which are excited via their phonon-side band, that are observed in the emission spectra through their ZPLs. This behaviour is clearly confirmed through the series of excitation and emission spectra shown in detail Figure 3 (right side. The $10 \mathrm{~K}$ excitation spectra associated with the long-wavelength tail of the emission band $(720 \mathrm{~nm})$ contains contributions from centres with ZPLs peaking at 694, 667 (strongest ZPL), 613 and $582 \mathrm{~nm}$. These ZPLs are mounted over an excitation broadband, which decreases below $550 \mathrm{~nm}$ according to the NPD absorption (Figure 1).

The excitation spectrum corresponding to the $694 \mathrm{~nm}$ ZPL emission contains same ZPLs with weaker intensity, being the broadband excitation around $600 \mathrm{~nm}$ relatively more intense than ZPLs' intensity. However, the excitation spectrum corresponding to the most prominent ZPL emission (green spectrum at $668 \mathrm{~nm}$ in Figure 3(b)) is the weakest one of the series - only ZPLs at 612 and $582 \mathrm{~nm}$ are weakly observed-. Thus, it suggests that the PL intensity associated with the $667 \mathrm{~nm}$ centre is mostly contained in its ZPL. This behaviour contrasts, for example, with observations in the $612 \mathrm{~nm} \mathrm{ZPL}$ centre, as the excitation into its ZPL yields other ZPLs emissions. Under excitation at $530 \mathrm{~nm}$, PL consists mainly of a broad band around $680 \mathrm{~nm}$, indicating that the NPD specific centres are hardly excited from this wavelength range. Clarifying the nature of the different PL centres found specifically in NPD can result extremely useful to explore other ways of producing new nanodiamond-based nitrogen-lean dyes [24].

\section{Raman spectrum: pressure sensor}

\section{Quantum confinement}

The NPD Raman spectra have been obtained by exciting at several wavelengths: 488,514 and $785 \mathrm{~nm}$. In all cases the characteristic stretching first-order phonon mode is observed at $1331.9 \mathrm{~cm}^{-1}$ together with a strong PL background, the intensity of which is largely attenuated by exciting at $785 \mathrm{~nm}$. Figure 2(b) shows the Raman spectra of NPDs together with the Raman spectra of CVD and NatD for comparison. All the Raman peaks can be well described by a Lorentzian profile, the fitting parameters of which are also given there. Both CVD and NatD have a $F W H M=2.7 \mathrm{~cm}^{-1}$ that broadens more than a factor 3 in NPDs -8.4 and $9.0 \mathrm{~cm}^{-1}$ for the two studied NPD samples-. We associate this broadening to the nanopolycrystalline structure, confining the phonon coherence to the crystalline size. On the basis of FWHM vs. nanoparticle size correlations established in nano CVD [25-27], it was empirically established for size larger than about $5 \mathrm{~nm}$, that FWHM is related to the particle size $L$ (in $\mathrm{nm}$ ), through the expression $[26,27]$ :

$$
F W H M=A+B / L
$$

with $A=2.99 \mathrm{~cm}^{-1}$ and $B=145.7 \mathrm{~cm}^{-1} \mathrm{~nm}$. Adapting the equation to the current instrumental response using the FWHM from CVD and NatD $\left(A=2.7 \mathrm{~cm}^{-1}\right)$, we obtain a NPD crystal size of $L=24 \mathrm{~nm}$, from the average NPD Raman $F W H M=8.7 \mathrm{~cm}^{-1}$. These figures compare well with typical crystal size measured by electron microscopy, where typical values around 10-30 $\mathrm{nm}$ are mostly found [2]. This result could be anticipated by peak 
profile analysis using the phonon-dispersion coefficients $\partial \omega(q) / \partial q$ of the optical branch along different reciprocal space directions [25-29]. For sizes above $10 \mathrm{~nm}$, quantum confinement effects can be estimated from the FWHM with Lorentzian profiles, considering that the optical-phonon dispersion averaged over the Brillouin zone is about $\partial \omega / \partial q=$ $30 \mathrm{~cm}^{-1} \mathrm{~nm}$ [25-27]. It means that an additional peak broadening of $6 \mathrm{~cm}^{-1}$, entirely due to quantum confinement effects, yields a phonon wavevector uncertainty of $\Delta q=0.2$ $\mathrm{nm}^{-1}$. In terms of crystal size, it implies $L=\pi / \Delta q=15 \mathrm{~nm}$, which is of the same order of the empirical estimate. It must be noted that peak broadening due to strain has not been considered as this effect is negligible in comparison to size effects according to Xray diffraction analysis [2].

\section{Raman shift pressure coefficient}

An important application of the diamond phonon frequency concerns its use as a pressure sensor. It has a relatively small pressure coefficient $\left(2.9 \mathrm{~cm}^{-1} \mathrm{GPa}^{-1}\right.$ for the $1332 \mathrm{~cm}^{-1}$ Raman peak [10]) in comparison to silicon $\left(5.6 \mathrm{~cm}^{-1} \mathrm{GPa}^{-1}\right.$ [30]) but similar to moissanite (2.39 $\mathrm{cm}^{-1} \mathrm{GPa}^{-1}$ for Raman peak at $976.4 \mathrm{~cm}^{-1}$; and $2.31 \mathrm{~cm}^{-1} \mathrm{GPa}^{-1}$ for $793.1 \mathrm{~cm}^{-1}$ [9]). Outmost, diamond has huge pressure stability enabling pressure determination up to Mbar pressures [12-18]. Nevertheless, some complications appear using diamond as a pressure sensor in a DAC. Firstly, transparent diamond chips inside the pressure chamber are more difficult to see than coloured or PL probes, and the Raman signal overlaps with that of the anvil below about $100 \mathrm{GPa}$. Except for very high pressures where both diamond anvil and NPD signals are decoupled, the Raman signal of the NPD sensor can be difficult to measure in DACs due to signal overlap (Figure 1(a)), with the NPD signal being much less intense than that of the diamond anvil. Secondly, pressure determination from the diamond anvil culet is even more complicated due to the deconvolution procedure employed to extract the Raman signal of the anvil culet out of the whole anvil signal, as well as the uncertainty in its pressure coefficient. Actually, it has been reported [15] that the pressure coefficient of the culet Raman frequency depends on the anvil geometry and other structural factors. This probably justifies why different pressure coefficients ranging from 1.7 to $2.6 \mathrm{~cm}^{-1} \mathrm{GPa}^{-1}$ - almost $50 \%$ variation - have been reported up to now [12-18]. This situation makes pressure determination from diamond anvils in DACs far from being a universal pressure sensor. It is particularly more serious in static Mbar experiments where small deviations of the calibration equation, and the way of selecting the spectral signature of the anvil make this method quite controversial.

Aiming at clarifying the pressure Raman scale, we have measured the variation of NPD micro-chips as pressure gauge in a DAC and a MAC. Interestingly, the brownish colour of NPD makes it easy the identification of NPD chips inside the anvil cell. Figure 4 shows also the pressure-induced Raman shift of NPD in a DAC and in a MAC. It must be noted the difficulty to extract the NPD Raman signal in a DAC, which is completely masked by the Raman signal of diamond anvil below about $14 \mathrm{GPa}$. However, the evolution of the NPD Raman peak is very well observed in MAC, given that Raman peaks of moissanite at 976.4 and $793.1 \mathrm{~cm}^{-1}$ are largely shifted from the NPD peak at $1332 \mathrm{~cm}^{-1}$. This situation can be visualized through the set of spectra of Figure 4, where the intensity axes illustrate the relative Raman intensity obtained in DAC and MAC. The variation of the NPD Raman frequency $\omega(P)$ in both DAC and MAC is shown in Figure 5. The measured $\omega(P)$ dependence for NPD loaded in a DAC below $14 \mathrm{GPa}$ deviates from the expected linear behaviour 
(a)

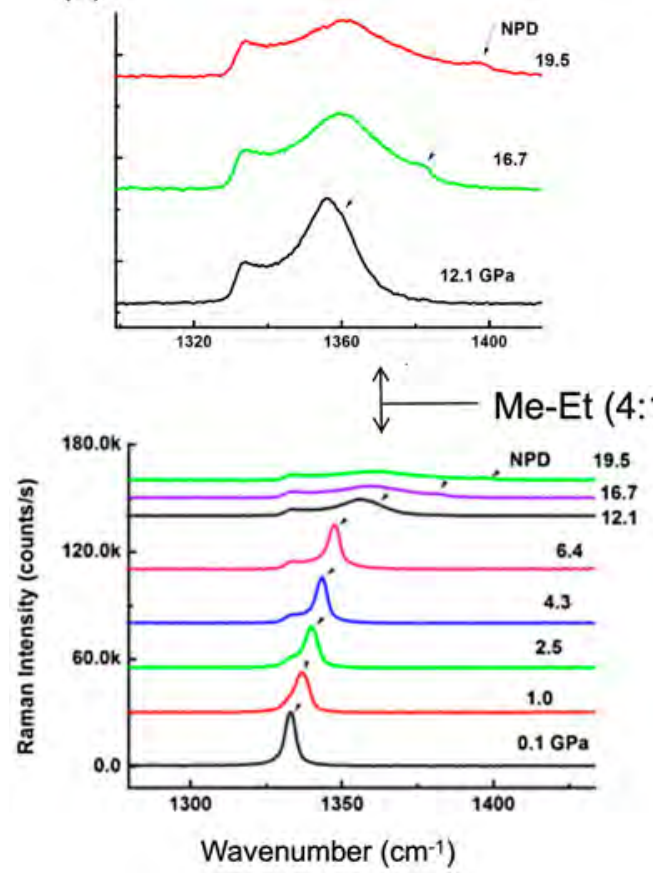

(b)

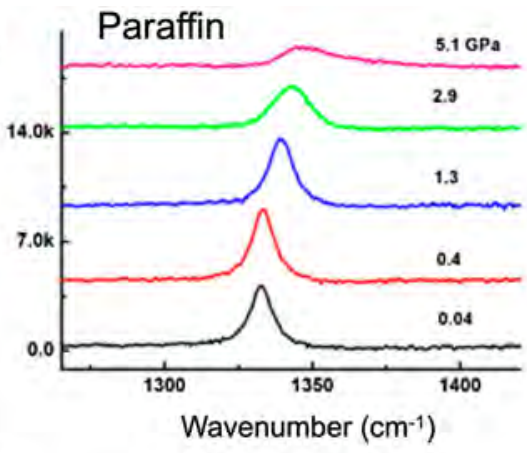

$10.0 \mathrm{k}$

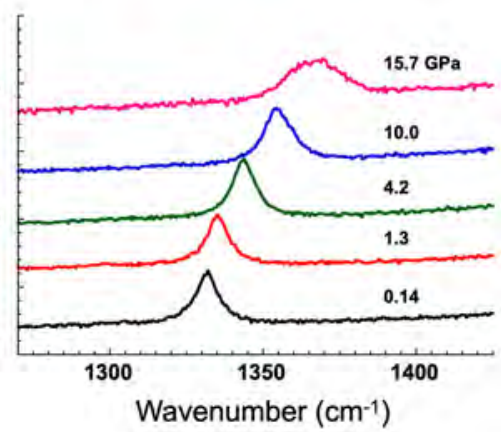

Figure 4. (a) Pressure dependence of the NPD Raman spectrum in a DAC using MeOH:EtOH (4:1) as pressure transmitting medium. Raman spectra are dominated by the anvil Raman signal. The small NPD Raman signal is marked with an arrow in each spectrum. Some high-pressure Raman spectra are magnified on top for illustrating the spectral shape. (b) NPD Raman spectra as a function of pressure in a MAC using spectroscopic paraffin oil - on top - and $\mathrm{MeOH}-\mathrm{EtOH}(4: 1)$ - at the bottom - as pressure transmitting media.

(a)

NPD in DAC

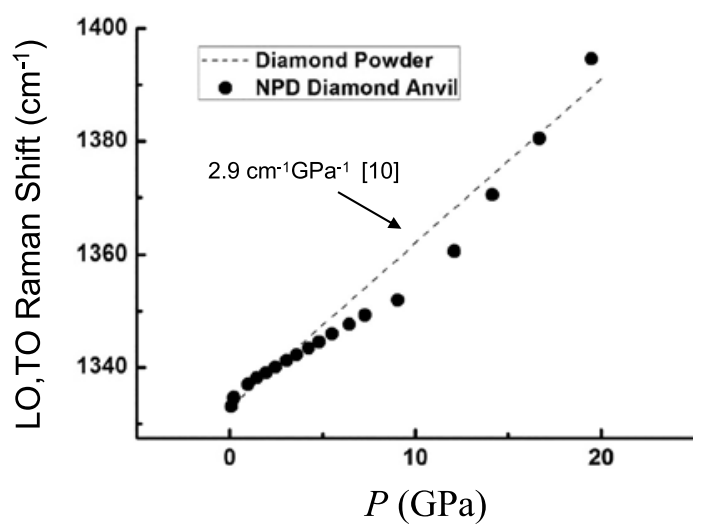

(b)

NPD in MAC

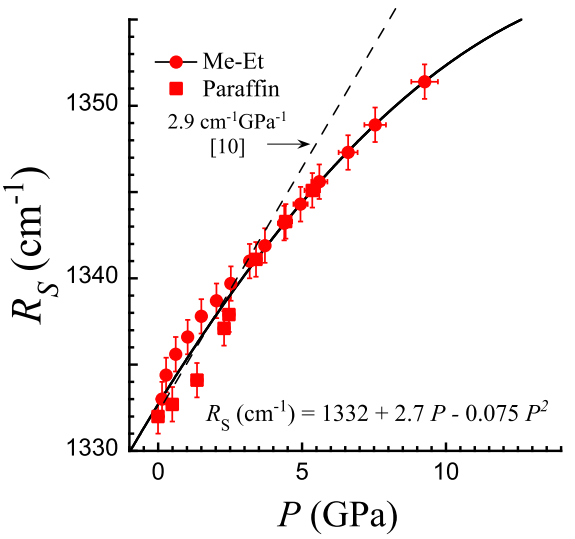

Figure 5. (a) Pressure dependence of NPD phonon frequency measured by Raman spectroscopy $\left(R_{S}\right)$ in a DAC using $\mathrm{MeOH}: \mathrm{EtOH}(4: 1)$. Results are compared with those reported for diamond powder elsewhere [10]. The lack of linearity in $R_{S}(P)-\omega(P)$ in the text - is due to extraction procedures to separate the NPD signal from the stronger NatD anvil signal. (b) Variation of the NPD Raman frequency with pressure measured in a MAC using both $\mathrm{MeOH}: \mathrm{EtOH}$ (4:1) and spectroscopic paraffin oil as pressure transmitting media. 
attained in single-crystal diamond - NatD-. It illustrates the difficulties to extract accurate pressure data from either the diamond anvil culet, or from NPD chips inside a DAC, particularly, at low pressure given that signal is mostly masked by the asymmetric broadened anvil signal. The NPD $\omega(P)$ data can be clearly obtained by loading microNPD samples in a MAC using either paraffin oil or Methanol-Ethanol (4:1) as pressure transmitting medium. The obtained linear pressure coefficient is $2.7 \mathrm{~cm}^{-1} \mathrm{GPa}^{-1}$, although a quadratic component in $\omega(P)$ is observed as well (Figure $5(b)$ ). A more thoroughly experiment has been done using MAC containing both NatD and NPD together with several ruby spheres. It enables us to achieve a more suitable comparison study between FWHM and frequency pressure rates in both diamonds under identical pressure conditions (Figure $6(a, b))$. It turns out that the NatD $\omega(P)$ experiences a linear shift with pressure at a rate of $2.73(4) \mathrm{cm}^{-1} \mathrm{GPa}^{-1}$ whereas NPD $\omega(P)$ experiences a slight quadratic response with pressure, with a linear coefficient at zero pressure of $2.89(15) \mathrm{cm}^{-1} \mathrm{GPa}^{-1}$ in the $0-$ $18 \mathrm{GPa}$ range.

Interestingly, the NPD Raman peak slightly broadens with pressure at a rate of 0.15 (2) $\mathrm{cm}^{-1} \mathrm{GPa}^{-1}$ under hydrostatic conditions. This behaviour contrasts with the ruby R-

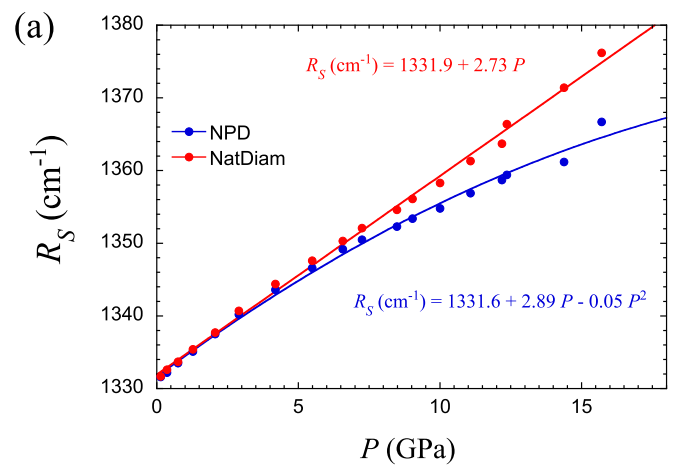

(c)

(b)
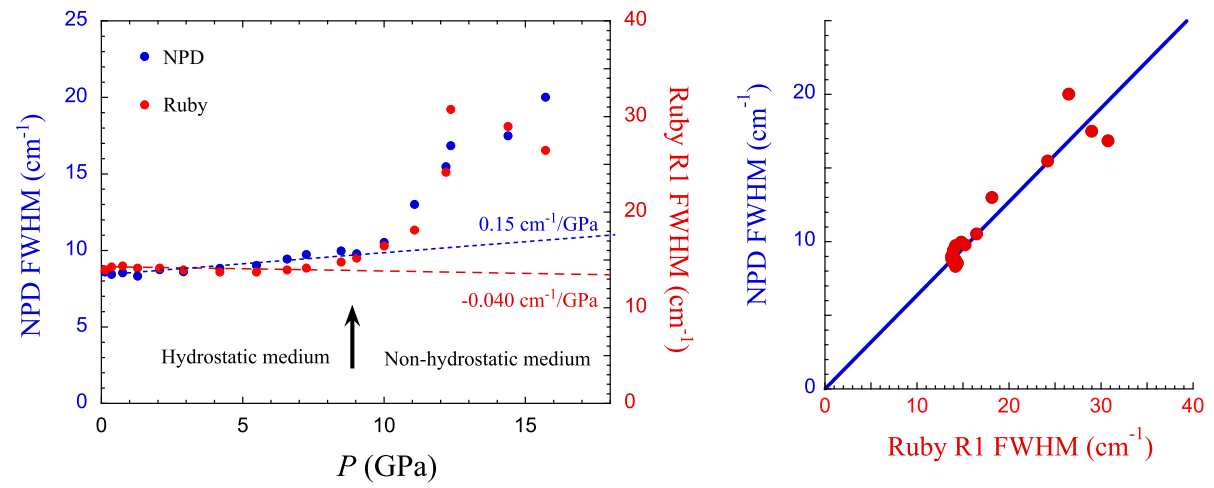

Figure 6. Plots of the pressure dependence of the NPD and NatD Raman frequency $R_{S}(P)-\omega(P)$ in the text - (a) and corresponding FWHM of the NPD Raman peak vs. the FWHM of the ruby $R_{1}$ emission peak (b) obtained in a MAC using $\mathrm{MeOH}-\mathrm{EtOH}(4: 1)$ as pressure transmitting medium in both hydrostatic $(P$ $<9 \mathrm{GPa})$ and non-hydrostatic $(P>9 \mathrm{GPa})$ regimes. (c) Image of the MAC cavity loaded with one NPD chip and ruby spheres at $6.4 \mathrm{GPa}$ (on top). Correlation between the FWHM of NPD Raman peak and ruby $R_{1}$ emission peak from the pressure experiment in a MAC using $\mathrm{MeOH}-\mathrm{EtOH}$ (4:1). A similar result was obtained using paraffin oil as pressure transmitting medium (at the bottom). 
lines emission, which FWHM slightly narrows with pressure $\left(-0.040(10) \mathrm{cm}^{-1} \mathrm{GPa}^{-1}\right)$ due mainly to hardening of acoustic mode frequency with pressure, yielding a reduction of the Stokes and anti-Stokes Raman processes involving acoustic modes. These processes explain why the FWHM of ZPL electronic transitions reduces with increasing pressure under isostatic conditions in ruby R-lines and other ZPLs involving $\mathrm{Ce}^{3+}[31,32]$.

Nevertheless, it must be pointed out that although these narrowing processes hold also for the Raman peak, there is an additional main contribution to the pressure-induced broadening due to an increase of decaying probability of LO and TO modes into acoustic phonons yielding lifetime reduction and a concomitant broadening of the phonon linewidth. This mechanism was first introduced by Ulrich et al. [33] to explain the pressureinduced broadening of the first-order Raman peak in $\mathrm{Si}, \mathrm{Ge}$, and $\mathrm{SiC}$ at low temperatures ( $T \sim 6 \mathrm{~K})$. Later Qiu et al. [34] measured the Raman line broadening by pressure in isotopically pure ${ }^{13} \mathrm{C}$ single-crystal diamond showing that the $F W H M(P)$ increased at a rate of $0.085 \mathrm{~cm}^{-1} \mathrm{GPa}^{-1}$ in the $0-120 \mathrm{GPa}$ range at room temperature. This figure is similar to those found in $\mathrm{Si}\left(0.137 \mathrm{~cm}^{-1} \mathrm{GPa}^{-1}\right)$ and $\mathrm{Ge}\left(0.037 \mathrm{~cm}^{-1} \mathrm{GPa}^{-1}\right)$ [33], and also to that measured in NPD in this work $\left(0.15 \mathrm{~cm}^{-1} \mathrm{GPa}^{-1}\right)$. Although an additional contribution due to nanocrystallite size reduction effects must be considered in NPD, it is very small compared to the phonon lifetime contribution. Taking the diamond bulk modulus of $K_{0}=444 \mathrm{GPa}$ [11], we estimate from Equation (1) a pressure-induced broadening of the Raman peak due to size effects of $0.005 \mathrm{~cm}^{-1} \mathrm{GPa}^{-1}$, which is an order of magnitude smaller than the measured values.

Interestingly, the Raman peak broadening effect in NPD is largely enhanced under nonhydrostatic conditions due to strain-induced broadening (Figure $6(b, c)$ ). Actually, this inhomogeneous broadening is similar to that found in the ruby emission lines at the onset of the hydrostaticity loss of the pressure transmitting medium as it has been confirmed for various media [31]. The pressure dependence of the Raman FWHM in both pressure transmitting media - paraffin oil and $\mathrm{MeOH}-\mathrm{EtOH}$ - and its comparison with the corresponding $R_{1}$-line FWHM, indicates that the NPD Raman FWHM is a good sensor to probe hydrostaticity of pressure transmitting media. Figure $6(b, c)$ shows the pressure dependence of NPD Raman FWHM and its variation with respect to the ruby $\mathrm{R}_{1}$-line $F W H M$, respectively. Both NPD-Raman and ruby- $R_{1}$-line $F W H M s$ are proportional to each other irrespective of the pressure transmitting media, and pressure regime as being hydrostatic or non-hydrostatic. The obtained relation, $\left[F W H M-F W H M_{0}\right](N P D)=$ 0.64 [FWHM-FWHM $M_{0}$ (ruby) with $F W H M_{0}$ being the ambient pressure values of $9 \mathrm{~cm}^{-1}$ (NPD) and $14 \mathrm{~cm}^{-1}$ (ruby), indicates that peak broadening effects in NPD is as sensitive as ruby, considering that the FWHM ratio at ambient conditions is a factor 1.5 . It means that the broadening of NPD is as sensitive as ruby to detect non-hydrostatic effects.

\section{Conclusions}

We showed that NPD photoluminescence is mainly due to specific centres, which are probably located at the inter-grain region of the nanocrystallites. Although the structure of such centres still deserves clarification, its understanding may guide new routes for exploring nitrogen-free photoluminescent diamond dyes. We also demonstrated that NPD is a good visible probe for pressure determination in pressure cells. Its pressure dependence in the $0-18 \mathrm{GPa}$ range has been measured in a moissanite anvil cell, 
showing a significant non-linear dependence as $\omega(P)=1331.6+2.89 P-0.05 P^{2}$ (in $\mathrm{cm}^{-1}$ and GPa units), in comparison to the linear behaviour of the natural single-crystal diamond of $2.73 \mathrm{~cm}^{-1} \mathrm{GPa}^{-1}$. The non-linear dependence of $\omega(P)$ enables NPD as pressure gauge in DACs, given that its Raman signal decouples from the anvil signal above about $15 \mathrm{GPa}$. Finally, the FWHM of the NPD Raman peak is an efficient probe to explore nonhydrostatic effects in comparison to the broadening of ruby R-lines. In conclusion, NPD provides good sensing capabilities as pressure gauge through its Raman frequency, as well as for probing non-hydrostatic effects through $F W H M(P)$.

\section{Acknowledgements}

We acknowledge the PRIUS Collaborative Project (2013-2018) through which we obtained suitable NPD samples.

\section{Disclosure statement}

No potential conflict of interest was reported by the authors.

\section{Funding}

Financial support from Projects MAT2015-69508-P; PGC2018-101464-B-I00 and MALTA -Consolider Team RED2018-102612-T (Ministerio de Ciencia, Innovación y Universidades) is acknowledged.

\section{References}

[1] Irifune T, Kurio A, Sakamoto $S$, et al. Ultrahard polycrystalline diamond from graphite. Nature. 2003;421:599-600.

[2] Sumiya $H$, Irifune T. Microstructure and mechanical properties of high- hardness nano-polycrystalline diamonds. SEl Tech Rev. 2008;66:85-91.

[3] Ishimatsu N, Kawamura N, Mizumaki M, et al. Applications of nano-polycrystalline diamond anvils to X-ray absorption spectroscopy under high pressure. High Press Res. 2016;36:381-390.

[4] Ohfuji H, Okada T, Yagi T, et al. Application of nano-polycrystalline diamond to laser-heated diamond anvil cell experiments. High Press Res. 2010;30:142-150.

[5] Sakai T, Yagi T, Ohfuji $\mathrm{H}$, et al. High-pressure generation using double stage micro-paired diamond anvils shaped by focused ion beam. Rev Sci Instr. 2015;86:033905-033905-6.

[6] Skalwold EA, Renfro N, Shigley JE, et al. Characterization of a synthetic nano-polycrystalline diamond gemstone. Gems Gemol. 2012;48:188-192.

[7] Sumiya H, Harano K, Arimoto K, et al. Optical characteristics of nano-polycrystalline diamond synthesized directly from graphite under high pressure and high temperature. Jpn J Appl Phys. 2009;48:120206-120206-3.

[8] Ikeda K, Sumiya H. Optical properties of ultrapure nano-polycrystalline diamond. Jpn J Appl Phys. 2016;55:120306-120306-3.

[9] Liu Z, Xu J, Scott HP. Moissanite ( $\mathrm{SiC}$ ) as windows and anvils for high-pressure infrared spectroscopy. Rev Sci Inst. 2004;75:5026-5029.

[10] Hanfland M, Syassen K. A Raman study of diamond anvils under stress. J Appl Phys. 1984;57:2752-2756.

[11] Aleksandrov IV, Goncharov AF, Zisma AN, et al. Diamond at high pressures: Raman scattering of light, equation of state, and high-pressure scale. Sov Phys JEPT. 1987;66:384-390.

[12] Occelli F, Loubeyere P, LeToullec R. Properties of diamond under hydrostatic pressures up to 140 GPa. Nature Mat. 2003;2:151-154. 
[13] Eremets M. Megabar high-pressure cells for Raman measurements. J. Raman Spectrosc. 2003;34:515-518.

[14] Akahama Y, Kawamura H. Pressure calibration of diamond anvil Raman gauge to $310 \mathrm{GPa}$. J Appl Phys. 2006;100:043516-043516-4.

[15] Baer BJ, Chang ME, Evans WJ. Raman shift of stressed diamond anvils: pressure calibration and culet geometry dependence. J Appl Phys. 2008;104:034504-034504-4.

[16] Akahama Y, Kawamura H. Pressure calibration of diamond anvil Raman gauge to $410 \mathrm{GPa}$. J Phys: Conf Ser. 2010;215:012195-012195-5.

[17] Ono S, Mibe K, Ohishi Y. Raman spectra of culet face of diamond anvils and application as optical pressure sensor to high temperatures. J Appl Phys. 2014;116:053517-053517-5.

[18] Fujii T, Ohfuji H. Pressure estimation using the 'diamond Raman scale' at low pressures in diamond anvil cell experiments using a highly confocal Raman system. Meas Sci Technol. 2015;26:025501-025501-5.

[19] Dischler B. Handbook of spectral lines in diamond, volume 1: tables and interpretations. Heidelberg: Springer-Verlag; 2012.

[20] Meng Y, Yan C, Lai J, et al. Enhanced optical properties of chemical vapor deposited single crystal diamond by low-pressure/high-temperature annealing. PNAS. 2008;105:17620-17625.

[21] Khan RUA, Martineau PM, Cann BL, et al. Charge transfer effects, thermo- and photochromism in single crystal CVD synthetic diamond. J Phys: Condens Matter. 2009;21:364214-364214-9.

[22] Wu BR. Structural and vibrational properties of the 6Hdiamond: first-principles study. Diamond Relat Mater. 2007;16:21-28.

[23] Karaveli S, Gaathon O, Wolcott A, et al. Modulation of nitrogen vacancy charge state and fluorescence in nanodiamonds using electrochemical potential. PNAS. 2016;113(15):3938-3943.

[24] Lühmann T, Raatz N, John R, et al. Screening and engineering of colour centres in diamond. J Phys D: Appl Phys. 2018;51:483002-483002-24.

[25] Xie J, Chen SP, Tse JS, et al. High-pressure thermal expansion, bulk modulus, and phonon structure of diamond. Phys Rev B. 1999;60:9444-9449.

[26] Osswald S, Mochalin VN, Havel M. Phonon confinement effects in the Raman spectrum of nanodiamond. Phys Rev B. 2009;80:075419-075419-9.

[27] Yoshikawa M, Mori Y, Maegawa M, et al. Raman scattering from diamond particles. Appl Phys Lett. 1993;62:3114-3116.

[28] Richter H, Wang ZP, Ley L. The one Raman-spectrum in microcrystalline silicon. Sol State Comm. 1981;39:625-629.

[29] Gonzalez J, Marquina J, Rodriguez F, et al. Nanocrystals of $\mathrm{ZnO}$ formed by the hot isostatic pressure method. High Press Res. 2009;29:594-599.

[30] García-Revilla S, Rodríguez $\mathrm{F}$, Valiente $\mathrm{R}$, et al. Optical spectroscopy of $\mathrm{Al}_{2} \mathrm{O}_{3}: \mathrm{Ti}^{3+}$ single crystal under hydrostatic pressure. the influence on the Jahn-Teller coupling. J Phys: Condens Matter. 2002;14:447-460.

[31] Klotz S, Chervin JC, Munsch P, et al. Hydrostatic limits of 11 pressure trans-mitting media. J Phys. D: Appl Phys. 2009;42:075413-075413-7.

[32] Valiente R, Rodríguez F, González J, et al. High pressure optical spectroscopy of $\mathrm{Ce}^{3+}$-doped $\mathrm{Cs}_{2} \mathrm{NaLuCl}_{6}$. Chem Phys Lett. 2009;481:149-151.

[33] Ulrich C, Anastassakis E, Syassen K, et al. Lifetime of phonons in semiconductors under pressure. Phys. Rev. Lett. 1997;78:1283.

[34] Qiu W, Velisavljevic N, Baker PA, et al. Isotopically pure ${ }^{13} \mathrm{C}$ layer as a stress sensor in a diamond anvil cell. Appl. Phys. Lett. 2004;84:5308. 